\title{
Quiver polynomials in iterated residue form
}

\author{
R. Rimányi
}

Received: 21 February 2013 / Accepted: 20 December 2013 / Published online: 14 January 2014

(C) Springer Science+Business Media New York 2014

\begin{abstract}
Degeneracy loci polynomials for quiver representations generalize several important polynomials in algebraic combinatorics. In this paper we give a nonconventional generating sequence description of these polynomials when the quiver is of Dynkin type.
\end{abstract}

Keywords Quiver · Degeneracy loci · Equivariant cohomology · Iterated residues

\section{Introduction}

Let us associate nonnegative integers to the vertices of an oriented graph. This combinatorial data determines a so-called quiver representation of a group $\Gamma$ on a vector space $V$. If the underlying unoriented graph is of simply laced Dynkin type, then the quiver representation has finitely many orbits. Let $\Omega$ be one of these orbits. The main object of this paper is the equivariant fundamental class

$$
[\bar{\Omega}] \in H_{\Gamma}^{*}(V) .
$$

The ring $H_{\Gamma}^{*}(V)$ is a polynomial ring, hence one calls the class $[\bar{\Omega}]$ a quiver polynomial.

Tracing back the definitions of equivariant cohomology we obtain that quiver polynomials are universal polynomials representing degeneracy loci. We will illustrate this statement with the following example. Let $\mathcal{E}_{1}$ and $\mathcal{E}_{2}$ be bundles of rank $e_{1}$ and $e_{2}$ over the compact complex manifold $X$, and let $\phi$ be a bundle map $\mathcal{E}_{1} \rightarrow \mathcal{E}_{2}$ which

R. Rimányi (凶)

Department of Mathematics, University of North Carolina at Chapel Hill, Chapel Hill, USA

e-mail: rimanyi@email.unc.edu

R. Rimányi

Department of Mathematics, University of Geneva, Geneva, Switzerland 
satisfies a certain transversality condition. Let $r \leq e_{1} \leq e_{2}$, and define the degeneracy locus

$$
\Omega_{r}(\phi)=\left\{x \in X: \operatorname{rk}\left(\phi_{x}:\left(\mathcal{E}_{1}\right)_{x} \rightarrow\left(\mathcal{E}_{2}\right)_{x}\right) \leq e_{1}-r\right\} .
$$

The Giambelli-Thom-Porteous formula claims that the fundamental cohomology class $\left[\Omega_{r}(\phi)\right] \in H^{*}(X)$ can be expressed as $\operatorname{det}\left(c_{r+j-i}\left(\mathcal{E}_{1}^{*}-\mathcal{E}_{2}^{*}\right)\right)_{i, j=1, \ldots, e_{2}-e_{1}+r}$. The polynomial $\operatorname{det}\left(c_{r+j-i}\right)_{i, j}$ is a quiver polynomial. It only depends on the combinatorial data $\bullet \rightarrow \bullet$ (the graph), $e_{1}, e_{2}$ (the ranks of the bundles), and $r$ (the orbit). Nevertheless, it has the universality property that after substituting $c_{i}\left(\mathcal{E}_{1}^{*}-\mathcal{E}_{2}^{*}\right)$ into the variables $c_{i}$, the polynomial expresses the fundamental class of the degeneracy locus $\Omega_{r}(\phi)$.

Quiver polynomials are analogous universal polynomials for more complicated degeneracy loci. This more complicated setting involves more than two vector bundles over $X$ (the vertices of the graph), with some bundle maps among them (the edges of the graph). The degeneracy locus is then defined by degenerations of a diagram of linear maps between vector spaces.

History Quiver polynomials of Dynkin type generalize several important polynomials in Algebraic Combinatorics, for example the Giambelli-Thom-Porteous formulas [28], the double Schur and Schubert polynomials of Schubert calculus [17], and the quantum [16] and universal Schubert polynomials [17].

There has been a lot of activity in the past decade or so to find various formulas and/or algorithms to calculate quiver polynomials of certain Dynkin types, such as $[4-10,12,16,24,25]$. By now we can claim that there are effective methods to find any such quiver polynomial. The three papers that approach the problem for general quivers of Dynkin type A, D, E are [12, 24] and [5].

The structure of quiver polynomials The key challenge of the theory is understanding the structure of quiver polynomials. The following two phenomena have been discovered/conjectured:

- Stability. In [14] the authors study an analogous problem: Thom polynomials of singularities. They find that equivariant fundamental classes display unexpected stability properties $[14$, Theorems $2.1,2.4]$, which enables them to organize infinitely many such classes into a (nonconventional) rational generating sequence. This phenomenon is further developed and organized in [2, 15, 23] (under the name of Iterated Residue generating sequence). Theorem 2.1 of [14] applies to quiver representations as well.

- Positivity. In [5] Buch proved that quiver polynomials are linear combinations of certain products of Schur polynomials. He conjectured that the coefficients in this expression are all nonnegative. These coefficients provide a wide generalization of several combinatorial constants in the style of Littlewood-Richardson coefficients.

In this paper we prove an Iterated Residue description of quiver polynomials. This description reduces Buch's conjecture to a positivity conjecture on the coefficients of certain expansions of rational functions. R. Kaliszewski [20] has promising initial results towards proving this algebraic positivity conjecture. 


\section{Combinatorics of polynomials}

\section{$2.1 \Delta$-Polynomials, Schur polynomials}

Let $c_{n}$ be elements of a ring for $n \in \mathbb{Z}$. Assume that $c_{0}=1$ and $c_{n}=0$ for $n<0$. Write $\mathbb{K}=\left(c_{n}\right)_{n \in \mathbb{Z}}$. Let $\lambda=\left(\lambda_{1}, \ldots, \lambda_{r}\right) \in \mathbb{Z}^{r}$ be an integer vector.

Definition 2.1 Define the $\Delta$-polynomial

$$
\Delta_{\lambda}=\Delta_{\lambda}(\mathbb{K})=\operatorname{det}\left(c_{\lambda_{i}+j-i}\right)_{i, j=1, \ldots, r} .
$$

If $\lambda$ is a partition, i.e. if $\lambda_{1} \geq \lambda_{2} \geq \cdots \geq \lambda_{r}$, then we call a $\Delta$-polynomial a Schur polynomial. It is known that if the underlying ring is $\mathbb{Z}\left[c_{1}, c_{2}, \ldots\right]$, then the Schur polynomials form an additive basis of this ring.

When we want to emphasize that a $\Delta$-polynomial is not a Schur polynomial, then we call it a fake Schur polynomial. Observe that a fake Schur polynomial is either 0, or is equal to plus or minus a Schur polynomial. For example $\Delta_{34}=0, \Delta_{14}=-\Delta_{32}$, $\Delta_{154}=\Delta_{433}$.

\subsection{The $\boldsymbol{C}$ and $\boldsymbol{\Delta}$-operations}

Let $p$ be a positive integer. For all $k=1, \ldots, p$ let $\mathbb{K}_{k}=\left(c_{k n}\right)_{n \in \mathbb{Z}}$ be a set of elements in a ring, as in Sect. 2.1. In particular, for all $k$ we have $c_{k 0}=1$ and $c_{k n}=0$ for $n<0$.

Let $\mathbb{A}_{1}, \ldots, \mathbb{A}_{p}\left(\left|\mathbb{A}_{k}\right|=r_{k}\right)$ be disjoint finite ordered sets of variables: $\mathbb{A}_{k}=$ $\left\{u_{k 1}, \ldots, u_{k r_{k}}\right\}$.

Definition 2.2 For a Laurent monomial in the variables $\bigcup_{k} \mathbb{A}_{k}$ define

$$
\boldsymbol{C}\left(\prod_{k=1}^{p} \prod_{s=1}^{r_{k}} u_{k s}^{\lambda_{k s}}\right)=\boldsymbol{C}_{\mathbb{A}_{1}, \ldots, \mathbb{A}_{p}}^{\mathbb{K}_{1}, \ldots, \mathbb{K}_{p}}\left(\prod_{k=1}^{p} \prod_{s=1}^{r_{k}} u_{k s}^{\lambda_{k s}}\right)=\prod_{k=1}^{p} \prod_{s=1}^{r_{k}} c_{k \lambda_{k s}} .
$$

For an element of $\mathbb{Z}\left[\left[u_{k s}^{ \pm 1}\right]\right]$, which has finitely many monomials with non-0 $\boldsymbol{C}$-value, extend this operation linearly.

Definition 2.3 For a Laurent monomial in the variables $\bigcup_{k} \mathbb{A}_{k}$ define

$$
\boldsymbol{\Delta}\left(\prod_{k=1}^{p} \prod_{s=1}^{r_{j}} u_{k s}^{\lambda_{k s}}\right)=\boldsymbol{\Delta}_{\mathbb{A}_{1}, \ldots, \mathbb{A}_{p}}^{\mathbb{K}_{1}, \ldots, \mathbb{K}_{p}}\left(\prod_{k=1}^{p} \prod_{s=1}^{r_{j}} u_{k s}^{\lambda_{k s}}\right)=\prod_{k=1}^{p} \Delta_{\lambda_{k 1}, \ldots, \lambda_{k r_{k}}}\left(\mathbb{K}_{k}\right) .
$$

For an element of $\mathbb{Z}\left[\left[u_{k s}^{ \pm 1}\right]\right]$, which has finitely many monomials with non-0 $\boldsymbol{\Delta}$-value, extend this operation linearly.

Example 2.4 Let $\mathbb{A}_{1}=\left\{u_{1}, u_{2}\right\}, \mathbb{A}_{2}=\left\{v_{1}, v_{2}\right\}, \mathbb{A}_{3}=\{w\}$, and let $\mathbb{K}_{1}=\left(c_{n}\right), \mathbb{K}_{2}=$ $\mathbb{K}_{3}=\left(d_{n}\right)$. Here are some examples for the $\boldsymbol{C}$ and $\boldsymbol{\Delta}$-operations. 
- $\boldsymbol{C}\left(u_{1}^{2} u_{2}^{1} v_{1}^{5} v_{2}^{1} w^{2}+u_{1}^{2} u_{2}^{1} v_{1}^{1} v_{2}^{2} w^{3}+u_{1}^{1} u_{2}^{4}+v_{1}^{-1} v_{2}^{2}+u_{1}^{-1} u_{2}^{-2}\right)=c_{2} c_{1} d_{5} d_{1} d_{2}+$ $c_{2} c_{1} d_{1} d_{2} d_{3}+c_{1} c_{4}+\underbrace{d_{-1}}_{0} d_{2}+\underbrace{c_{-1}}_{0} \underbrace{c_{-2}}_{0}$.

- $\boldsymbol{C}\left(u_{1}^{2} u_{2}^{2}\left(u_{1}-u_{2}\right)\right)=\boldsymbol{C}\left(u_{1}^{3} u_{2}^{2}-u_{1}^{2} u_{2}^{3}\right)=c_{3} c_{2}-c_{2} c_{3}=0$.

- $\boldsymbol{C}\left(\frac{u_{1} u_{2}}{1-\frac{u_{1}}{u_{2}}}\right)=\boldsymbol{C}\left(u_{1} u_{2}+u_{1}^{2}+u_{1}^{3} u_{2}^{-1}+\cdots\right)=c_{1}^{2}+c_{2}$.

- $\boldsymbol{\Delta}\left(u_{1}^{2} u_{2}^{1} v_{1}^{5} v_{2}^{1} w^{2}+u_{1}^{2} u_{2}^{1} v_{1}^{1} v_{2}^{2} w^{3}+u_{1}^{1} u_{2}^{4}+v_{1}^{-1} v_{2}^{2}+u_{1}^{-1} u_{2}^{-2}\right)=\Delta_{21}\left(\mathbb{K}_{1}\right) \Delta_{51}\left(\mathbb{K}_{2}\right) \times$ $\Delta_{2}\left(\mathbb{K}_{2}\right)+\Delta_{21}\left(\mathbb{K}_{1}\right) \underbrace{\Delta_{12}\left(\mathbb{K}_{2}\right)}_{0} \Delta_{3}\left(\mathbb{K}_{2}\right)+\underbrace{\Delta_{14}\left(\mathbb{K}_{1}\right)}_{-\Delta_{32}\left(\mathbb{K}_{1}\right)}+\underbrace{\Delta_{-1,2}\left(\mathbb{K}_{2}\right)}_{-\Delta_{10}\left(\mathbb{K}_{2}\right)}+\underbrace{\Delta_{-1,-2}\left(\mathbb{K}_{1}\right)}_{0}$.

- $\boldsymbol{\Delta}\left(\frac{u_{1} u_{2}}{1-\frac{u_{1}}{u_{2}}}\right)=\boldsymbol{\Delta}\left(u_{1} u_{2}+u_{1}^{2}+u_{1}^{3} u_{2}^{-1}+\cdots\right)=\Delta_{11}\left(\mathbb{K}_{1}\right)+\Delta_{20}\left(\mathbb{K}_{1}\right)+\underbrace{\Delta_{3,-1}\left(\mathbb{K}_{1}\right)+\ldots}_{0}$

Here we used a convention, that we will keep using in the whole paper: by the rational function $1 /(1-x / y)$ we always mean its expansion in the $|x| \ll|y|$ range, that is, $1+(x / y)+(x / y)^{2}+\cdots$.

The two defined operations are related with each other.

Lemma 2.5 Let $\mathbb{A}=\left\{u_{1}, \ldots, u_{r}\right\}$, and let $f$ be a function in these variables. Then

$$
C_{\mathbb{A}}^{\mathbb{K}}\left(f \cdot \prod_{i<j}\left(1-\frac{u_{i}}{u_{j}}\right)\right)=\boldsymbol{\Delta}_{\mathbb{A}}^{\mathbb{K}}(f) .
$$

The proof is an application of simple properties of the determinant and the discriminant $\prod_{i<j}\left(1-u_{i} / u_{j}\right)$, illustrated by the following special case:

$$
\boldsymbol{C}\left(\left(u_{1}^{8} u_{2}^{5}\right)\left(1-u_{1} / u_{2}\right)\right)=\boldsymbol{C}\left(u_{1}^{8} u_{2}^{5}-u_{1}^{9} u_{2}^{4}\right)=c_{8} c_{5}-c_{9} c_{4}=\Delta_{85}=\boldsymbol{\Delta}\left(u_{1}^{8} u_{2}^{5}\right) .
$$

We leave details to the reader.

\subsection{Constant-term formula}

The following lemma is a reformulation of the definition of $\boldsymbol{C}$.

Lemma 2.6 Let $\mathbb{K}_{k}=\left(c_{k n}\right), \mathbb{A}_{k}=\left\{u_{k s}\right\}$ for $k=1, \ldots, p$. Then

$$
C_{\mathbb{A}_{1}, \ldots, \mathbb{A}_{p}}^{\mathbb{K}_{1}, \ldots, \mathbb{K}_{p}}\left(f\left(u_{k s}\right)\right)=\left\{f\left(u_{k s}\right) \cdot \prod_{k, s} \sum_{n=0}^{\infty} \frac{c_{k n}}{u_{k s}^{n}}\right\}_{u_{k s}},
$$

where \{\}$_{u_{k s}}$ means the constant coefficient in the $u_{k s}$ variables.

Remark 2.7 Taking the constant term (after shifting of exponents) is an iterated residue operation. Hence, we call $\boldsymbol{C}$ and $\boldsymbol{\Delta}$ Iterated Residue operations, cf. [2], [15, Sect. 11], [23]. 


\section{Quivers of bundles, and their characteristic classes}

Let $\mathcal{Q}=\left(\mathcal{Q}_{0}, \mathcal{Q}_{1}\right)$ be an oriented graph (the quiver), obtained by choosing an arbitrary orientation on the edges of one of the simply laced Dynkin diagrams $A_{N}, D_{N}, E_{6}, E_{7}, E_{8}$. (We will say that $\mathcal{Q}$ is of Dynkin type or that $\mathcal{Q}$ is a Dynkin graph.) Let $\mathcal{Q}_{0}=\{1,2, \ldots, N\}$ be the set of vertexes, and let $\mathcal{Q}_{1}$ be the set of arrows (oriented edges). An arrow $a \in \mathcal{Q}_{1}$ has a tail $t(a) \in \mathcal{Q}_{0}$ and a head $h(a) \in \mathcal{Q}_{0}$. For $i \in \mathcal{Q}_{0}$ define

$$
\begin{aligned}
& T(i)=\left\{j \in \mathcal{Q}_{0} \mid \exists a \in \mathcal{Q}_{1} \text { with } t(a)=j, h(a)=i\right\}, \\
& H(i)=\left\{j \in \mathcal{Q}_{0} \mid \exists a \in \mathcal{Q}_{1} \text { with } h(a)=j, t(a)=i\right\} .
\end{aligned}
$$

By a $\mathcal{Q}$-bundle over a space $X$ we mean a collection of vector bundles $\mathcal{E}_{i}, i \in \mathcal{Q}_{0}$. A characteristic class of a $\mathcal{Q}$-bundle is a polynomial in the Chern classes of the bundles $\mathcal{E}_{1}, \ldots, \mathcal{E}_{N}$.

Let $\mathcal{M}_{i}=\bigoplus_{j \in T(i)} \mathcal{E}_{j}$, and consider the virtual bundle $\mathcal{M}_{i}^{*}-\mathcal{E}_{i}^{*}$, where ${ }^{*}$ means the dual bundle. Its Chern classes are defined by the formal expansion

$$
\sum_{n=0}^{\infty} c_{n}\left(\mathcal{M}_{i}^{*}-\mathcal{E}_{i}^{*}\right) \xi^{n}=\frac{\sum_{n=0}^{\mathrm{rk} \mathcal{M}_{i}} c_{n}\left(\mathcal{M}_{i}\right)(-\xi)^{n}}{\sum_{n=0}^{\mathrm{rk} \mathcal{E}_{i}} c_{n}\left(\mathcal{E}_{i}\right)(-\xi)^{n}}=\frac{\prod_{j \in T(i)}\left(\sum_{n=0}^{\mathrm{rk} \mathcal{E}_{j}} c_{n}\left(\mathcal{E}_{j}\right)(-\xi)^{n}\right)}{\sum_{n=0}^{\mathrm{rk} \mathcal{E}_{i}} c_{n}\left(\mathcal{E}_{i}\right)(-\xi)^{n}}
$$

Let $\mathbb{K}_{i}=\left(c_{n}\left(\mathcal{M}_{i}^{*}-\mathcal{E}_{i}^{*}\right)\right)_{n}$. We will be concerned with characteristic classes of a $\mathcal{Q}$-bundle that can be written as polynomials in $\bigcup \mathbb{K}_{i}$. For example, suppose we have sets of variables

$$
\mathbb{A}_{1}=\left\{u_{11}, \ldots, u_{1 r_{1}}\right\}, \quad \ldots, \quad \mathbb{A}_{p}=\left\{u_{p 1}, \ldots, u_{p r_{p}}\right\},
$$

and numbers $i_{1}, \ldots, i_{p} \in \mathcal{Q}_{0}$. Then for any polynomial (or Laurent polynomial) $f$ in the variables $u_{k s}$, the expressions

$$
\boldsymbol{C}_{\mathbb{A}_{1}, \ldots, \mathbb{A}_{p}}^{\mathbb{K}_{i_{1}}, \ldots, \mathbb{K}_{i_{p}}}(f), \quad \boldsymbol{\Delta}_{\mathbb{A}_{1}, \ldots, \mathbb{A}_{p}}^{\mathbb{K}_{i_{1}}, \ldots, \mathbb{K}_{i_{p}}}(f)
$$

are characteristic classes of the $\mathcal{Q}$-bundle.

\section{Quiver representations, quiver polynomials}

\subsection{Quiver representations, and their orbits}

Recall that $\mathcal{Q}=\left(\mathcal{Q}_{0}, \mathcal{Q}_{1}\right)$ is an oriented Dynkin graph, with the set $\mathcal{Q}_{0}=\{1,2, \ldots, N\}$ of vertices, and the set $\mathcal{Q}_{1}$ of arrows $a=(t(a), h(a)) \in \mathcal{Q}_{0}^{2}$. Fix a dimension vector $\left(e_{1}, \ldots, e_{N}\right) \in \mathbb{N}^{N}$, and vector spaces $E_{i}=\mathbb{C}^{e_{i}}$. We define the quiver representation of the group $\Gamma=\times_{i=1}^{N} G L\left(E_{i}\right)$ on the vector space $V=\bigoplus_{a \in \mathcal{Q}_{1}} \operatorname{Hom}\left(E_{t(a)}, E_{h(a)}\right)$ by

$$
\left(g_{i}\right)_{i \in \mathcal{Q}_{0}} \cdot\left(\phi_{a}\right)_{a \in \mathcal{Q}_{1}}=\left(g_{h(a)} \circ \phi_{a} \circ g_{t(a)}^{-1}\right)_{a \in \mathcal{Q}_{1}} .
$$


Since the underlying unoriented graph is of simply laced Dynkin type, the quiver representation has finitely many orbits [3] — for any dimension vector. Moreover, the list of orbits has an explicit description, as follows. Consider the set $\Phi^{+}$of positive roots, and the set $\left\{\alpha_{i}: i=1, \ldots, N\right\}$ of simple roots for the corresponding root system. For a positive root $\beta$ define $d_{i}(\beta)$ by $\beta=\sum_{i=1}^{N} d_{i}(\beta) \alpha_{i}$. The orbits of the quiver representation with Dynkin graph $\mathcal{Q}$ and dimension vector $\left(e_{1}, \ldots, e_{N}\right) \in \mathbb{N}^{N}$ are in one to one correspondence with vectors

$$
\left(m_{\beta}\right) \in \mathbb{N}^{\Phi^{+}}, \quad \text { for which } \sum_{\beta \in \Phi^{+}} m_{\beta} d_{i}(\beta)=e_{i} \quad \text { for all } i=1, \ldots, N \text {. }
$$

Note that the indexing set for the list of orbits does not depend on the orientation of $\mathcal{Q}$. The orbit corresponding to the vector $m=\left(m_{\beta}\right) \in \mathbb{N}^{\Phi^{+}}$will be denoted by $\Omega_{m}$.

\subsection{Quiver polynomials}

The main object of this paper is the $\Gamma$-equivariant cohomology class represented by the orbit closure $\bar{\Omega}_{m}$ in $V=\bigoplus_{a \in \mathcal{Q}_{1}} \operatorname{Hom}\left(E_{t(a)}, E_{h(a)}\right)$, i.e.

$$
\left[\bar{\Omega}_{m}\right] \in H_{\Gamma}^{*}(V) .
$$

There are several equivalent ways to define the equivariant class represented by an invariant subvariety in a representation, see for example [11, 13, 21], [27, 8.5], [18]. Formally we follow the treatment in [5], which defines the K-theory class of the subvariety, and defines the cohomology class as a certain leading coefficient.

The ring $H_{\Gamma}^{*}(V)$ is the ordinary cohomology ring $H^{*}\left(B_{\Gamma} V\right)$ of the so-called Borel-construction space $B_{\Gamma} V$. There is a natural vector bundle $B_{\Gamma} V \rightarrow B \Gamma$ with fiber $V$; where $B \Gamma$ is the classifying space of $\Gamma$. Hence $H_{\Gamma}^{*}(V) \cong H^{*}(B \Gamma)$, the ring of $\Gamma$-characteristic classes. Recall that $B \Gamma=B G L\left(E_{1}\right) \times \cdots \times B G L\left(E_{n}\right)$ and that over each $B G L\left(E_{i}\right)$ there is a natural tautological vector bundle $\mathcal{E}_{i}$ with fiber $E_{i}$. Consider the bundles $\mathcal{E}_{i}$ over $B \Gamma$. This way we obtained a $\mathcal{Q}$-bundle over $B \Gamma$. Recall that $\mathcal{M}_{i}=\bigoplus_{j \in T(i)} \mathcal{E}_{i}$, and define

$$
\mathbb{K}_{i}=\left(c_{n}\left(\mathcal{M}_{i}^{*}-\mathcal{E}_{i}^{*}\right)\right)_{n} .
$$

Our goal is to express the class $\left[\bar{\Omega}_{m}\right]$ in terms of the characteristic classes $\bigcup \mathbb{K}_{i}$.

\section{The main result}

\subsection{Resolution pair}

First we follow Reineke [29] to define resolution pairs, see also [5]. For dimension vectors $e, f \in \mathbb{N}^{N}$ let $\langle e, f\rangle=\sum_{i=1}^{N} e_{i} f_{i}-\sum_{a \in \mathcal{Q}_{1}} e_{t(a)} f_{h(a)}$ denote the Euler form for $\mathcal{Q}$. Identifying a positive $\operatorname{root} \beta$ with its dimension vector $d(\beta) \in \mathbb{N}^{N}$ by $\beta=$ $\sum_{i=1}^{N} d_{i}(\beta) \alpha_{i}$ (where $\alpha_{i}$ are the simple roots), we can extend the Euler form for positive roots. 
Let $\Phi^{\prime} \subset \Phi^{+}$be a subset of the set of positive roots. A partition

$$
\Phi^{\prime}=\mathcal{I}_{1} \cup \mathcal{I}_{2} \cup \cdots \cup \mathcal{I}_{S}
$$

is called directed if $\left\langle\beta_{1}, \beta_{2}\right\rangle \geq 0$ for all $\beta_{1}, \beta_{2} \in \mathcal{I}_{j}(1 \leq j \leq s)$, and $\left\langle\beta_{1}, \beta_{2}\right\rangle \geq 0 \geq$ $\left\langle\beta_{2}, \beta_{1}\right\rangle$ for all $\beta_{1} \in \mathcal{I}_{i}, \beta_{2} \in \mathcal{I}_{j}$ with $1 \leq i<j \leq s$. A directed partition exists for any Dynkin quiver, see [29].

Let $m=\left(m_{\beta}\right) \in \mathbb{N}^{\Phi^{+}}$be a vector representing an orbit $\Omega_{m} \subset V$, let $\Phi^{\prime} \subset \Phi^{+}$be a subset containing $\left\{\beta: m_{\beta} \neq 0\right\}$, and let $\Phi^{\prime}=\mathcal{I}_{1} \cup \cdots \cup \mathcal{I}_{s}$ be a directed partition. For each $j \in\{1, \ldots, s\}$ write

$$
\sum_{\beta \in \mathcal{I}_{j}} m_{\beta} \beta=\left(p_{1}^{(j)}, \ldots, p_{N}^{(j)}\right) \in \mathbb{N}^{N}
$$

Let $\boldsymbol{i}^{(j)}=\left(i_{1}, \ldots, i_{l}\right)$ be any sequence of the vertices $i \in \mathcal{Q}_{0}$ for which $p_{i}^{(j)} \neq 0$, with no vertices repeated, and ordered so that the tail of any arrow of $\mathcal{Q}$ comes before its head. Let $\boldsymbol{r}^{(j)}=\left(p_{i_{1}}^{(j)}, \ldots, p_{i_{l}}^{(j)}\right)$. Finally, let $\boldsymbol{i}$ and $\boldsymbol{r}$ be the concatenated sequences $\boldsymbol{i}=\boldsymbol{i}^{(1)} \boldsymbol{i}^{(2)} \ldots \boldsymbol{i}^{(s)}$ and $\boldsymbol{r}=\boldsymbol{r}^{(1)} \boldsymbol{r}^{(2)} \ldots \boldsymbol{r}^{(s)}$. The pair $\boldsymbol{i}, \boldsymbol{r}$ will be called a resolution pair for $\Omega_{m}$.

5.2 Resolution variables, generating functions

For the resolution pair

$$
\boldsymbol{i}=\left(i_{1}, \ldots, i_{p}\right), \quad \boldsymbol{r}=\left(r_{1}, \ldots, r_{p}\right)
$$

we define the sets of variables $\mathbb{A}_{k}=\left\{u_{k 1}, \ldots, u_{k r_{k}}\right\}$ for $k=1, \ldots, p$. Set

$$
\mathbb{B}_{k}=\bigcup_{\substack{l>k \\ i_{l}=i_{k}}} \mathbb{A}_{l}, \quad \mathbb{C}_{k}=\bigcup_{\substack{l>k \\ i_{l} \in H\left(i_{k}\right)}} \mathbb{A}_{l}, \quad n_{k}=\sum_{\substack{l>k \\ i_{l} \in T\left(i_{k}\right)}} r_{l}-\sum_{\substack{l>k \\ i_{l}=i_{k}}} r_{l} .
$$

Next we define various functions in the variables $\bigcup_{k} \mathbb{A}_{k}$. For $1 \leq k \leq p$, define:

- the monomial factors

$$
M_{k}=\prod_{s=1}^{r_{k}} u_{k s}^{n_{k}}
$$

- the discriminant factors

$$
D_{k}=\prod_{1 \leq i<j \leq r_{k}}\left(1-\frac{u_{k i}}{u_{k j}}\right)
$$

- the interference factors

$$
I_{k}=\prod_{s=1}^{r_{k}} \frac{\prod_{x \in \mathbb{B}_{k}}\left(1-\frac{u_{k s}}{x}\right)}{\prod_{x \in \mathbb{C}_{k}}\left(1-\frac{u_{k s}}{x}\right)}
$$


Theorem 5.1 Recall the definition of $\mathbb{K}_{i}$ from (3). We have

$$
\begin{aligned}
& {\left[\bar{\Omega}_{m}\right]=C_{\mathbb{A}_{1}, \ldots, \mathbb{A}_{p}}^{\mathbb{K}_{i_{1}}, \ldots, \mathbb{K}_{i_{p}}}\left(\prod_{k=1}^{p} M_{k} D_{k} I_{k}\right) ;} \\
& {\left[\bar{\Omega}_{m}\right]=\boldsymbol{\Delta}_{\mathbb{A}_{1}, \ldots, \mathbb{A}_{p}}^{\mathbb{K}_{i_{1}}, \ldots, \mathbb{K}_{i_{p}}}\left(\prod_{k=1}^{p} M_{k} I_{k}\right) .}
\end{aligned}
$$

The right-hand sides of (5) and (6) are equal, because of Lemma 2.5. We will prove (6) in Sect. 7.

Remark 5.2 The key ingredient of the above expressions is $\prod_{k} I_{k}$. It can be rewritten as

$$
\prod_{k=1}^{p} I_{k}=\frac{\prod_{v \in Q_{0}} \prod_{k<l, i_{k}=i_{l}=v} \quad \prod_{x \in \mathbb{A}_{k}, y \in \mathbb{A}_{l}}\left(1-\frac{x}{y}\right)}{\prod_{a \in Q_{1}} \prod_{k<l, i_{k}=t(a), i_{l}=h(a)} \prod_{x \in \mathbb{A}_{k}, y \in \mathbb{A}_{l}}\left(1-\frac{x}{y}\right)} .
$$

The resemblance of this formula with the one in Theorem 2 of [26] for the multiplication in the Cohomological Hall Algebra, is not accidental. The connection between COHA's and quiver polynomials is explained in [30].

Remark 5.3 Lemma 2.5 allows us to write (6) in another equivalent form, as follows. Let $\mathbb{X}_{i}$ be the concatenation of alphabets $\mathbb{A}_{j_{1}} \mathbb{A}_{j_{2}} \cdots \mathbb{A}_{j_{s}}$ with $j_{1}<j_{2}<\cdots<j_{s}$ and $i_{j_{1}}=\cdots=i_{j_{s}}=i$. Then

$$
\left[\bar{\Omega}_{m}\right]=\boldsymbol{\Delta}_{\mathbb{X}_{1}, \ldots, \mathbb{X}_{N}}^{\mathbb{K}_{1}, \ldots, \mathbb{K}_{N}}\left(\frac{\prod_{k=1}^{p} M_{k}}{\prod_{a \in Q_{1}} \prod_{k<l, i_{k}=t(a), i_{l}=h(a)} \prod_{x \in \mathbb{A}_{k}, y \in \mathbb{A}_{l}}\left(1-\frac{x}{y}\right)}\right) .
$$

\subsection{An $A_{3}$ example}

Consider the quiver $1 \rightarrow 2 \leftarrow 3$. The positive roots of the root system $A_{3}$ are $\beta_{i j}=\sum_{i \leq u \leq j} \alpha_{u}$ for $1 \leq i \leq j \leq 3$. Consider the orbit corresponding to the linear combination $\sum_{1 \leq i \leq j \leq 3} m_{i j} \beta_{i j}$. Let us choose the partition

$$
\Phi^{+}=\left\{\beta_{22}\right\} \cup\left\{\beta_{12}, \beta_{23}, \beta_{13}\right\} \cup\left\{\beta_{11}, \beta_{33}\right\} .
$$

This choice induces the resolution pair $\boldsymbol{i}=(2,1,3,2,1,3)$,

$$
\boldsymbol{r}=\left(m_{22}, m_{12}+m_{13}, m_{23}+m_{13}, m_{12}+m_{13}+m_{23}, m_{11}, m_{33}\right) .
$$

Let $\mathbb{A}_{i}, i=1, \ldots, 6$ be alphabets of cardinalities $r_{i}$ respectively. We have

$$
\begin{aligned}
& \mathbb{B}_{1}=\mathbb{A}_{4}, \quad \mathbb{B}_{2}=\mathbb{A}_{5}, \quad \mathbb{B}_{3}=\mathbb{A}_{6}, \quad \mathbb{B}_{4}=\emptyset, \quad \mathbb{B}_{5}=\emptyset, \quad \mathbb{B}_{6}=\emptyset, \\
& \mathbb{C}_{1}=\emptyset, \quad \mathbb{C}_{2}=\mathbb{A}_{4}, \quad \mathbb{C}_{3}=\mathbb{A}_{4}, \quad \mathbb{C}_{4}=\emptyset, \quad \mathbb{C}_{5}=\emptyset, \quad \mathbb{C}_{6}=\emptyset, \\
& n_{1}=m_{13}+m_{11}+m_{33}, \quad n_{2}=-m_{11}, \quad n_{3}=-m_{33}, \quad n_{4}=m_{11}+m_{33} \text {, } \\
& n_{5}=0, \quad n_{6}=0 \text {. }
\end{aligned}
$$


Hence, (6) in Theorem 5.1 gives

$$
\begin{aligned}
& {\left[\bar{\Omega}_{m}\right]=\boldsymbol{\Delta}_{\mathbb{A}_{1}, \mathbb{A}_{2}, \mathbb{A}_{3}, \mathbb{A}_{4}, \mathbb{A}_{5}, \mathbb{A}_{6}}^{\mathbb{K}_{2}, \mathbb{K}_{1}, \mathbb{K}_{3}, \mathbb{K}_{2}, \mathbb{K}_{1}, \mathbb{K}_{3}}} \\
& \left(\mathbb{A}_{1}^{m_{13}+m_{11}+m_{33}} \mathbb{A}_{2}^{-m_{11}} \mathbb{A}_{3}^{-m_{33}} \mathbb{A}_{4}^{m_{11}+m_{33}} \frac{\left(1-\frac{\mathbb{A}_{1}}{\mathbb{A}_{4}}\right)\left(1-\frac{\mathbb{A}_{2}}{\mathbb{A}_{5}}\right)\left(1-\frac{\mathbb{A}_{3}}{\mathbb{A}_{6}}\right)}{\left(1-\frac{\mathbb{A}_{2}}{\mathbb{A}_{4}}\right)\left(1-\frac{\mathbb{A}_{3}}{\mathbb{A}_{4}}\right)}\right) .
\end{aligned}
$$

Here we used the shorthand notation $\mathbb{X}^{m}=\prod_{x \in \mathbb{X}} x^{m}$ and $1-\mathbb{X} / \mathbb{Y}=$ $\prod_{x \in \mathbb{X}} \prod_{y \in \mathbb{Y}}(1-x / y)$. This formula can be rewritten in the form of Remark 5.3 to obtain

$$
\left.\left[\bar{\Omega}_{m}\right]=\boldsymbol{\Delta}_{\left(\mathbb{A}_{1} \mathbb{A}_{4}\right),\left(\mathbb{A}_{2} \mathbb{A}_{5}\right),\left(\mathbb{A}_{3} \mathbb{A}_{6}\right)}^{\mathbb{K}_{2},} \underset{\mathbb{K}_{1}, \quad \mathbb{K}_{3}^{m_{13}+m_{11}+m_{33}} \mathbb{A}_{2}^{-m_{11}} \mathbb{A}_{3}^{-m_{33}} \mathbb{A}_{4}^{m_{11}+m_{33}}}{\left(1-\frac{\mathbb{A}_{2}}{\mathbb{A}_{4}}\right)\left(1-\frac{\mathbb{A}_{3}}{\mathbb{A}_{4}}\right)}\right) .
$$

It is tempting to call the functions in the arguments "generating functions": One only has to change the exponents $m_{i j}$ to obtain the polynomials $\left[\bar{\Omega}_{m}\right.$ ] for different $m$ values. Nevertheless, the lengths of the alphabets $\mathbb{A}_{k}$ also depend on $m_{i j}$, and the operations $\boldsymbol{C}, \boldsymbol{\Delta}$ are rather delicate. Hence, one may call the arguments "iterated residue generating functions."

\section{Concrete calculations}

In this section we show how the formulas (5), (6) and (8) work in concrete cases, that is, how they produce formulas involving characteristic classes.

Consider the quiver $1 \rightarrow 2 \leftarrow 3$ of Sect. 5.3, and let $m_{13}=m_{12}=m_{22}=m_{33}=1$, $m_{11}=m_{23}=0$. That is, we consider the orbit in the representation space with dimension vector $(2,3,2)$, whose strand diagram (see [1]) is in the picture below.

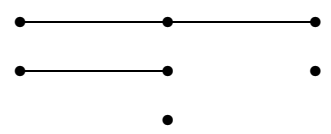

Again, choose the partition of positive roots as in Sect. 5.3. We obtain the following data:

$$
\begin{array}{llll}
\mathbb{A}_{1}=\{u\}, & \mathbb{A}_{2}=\left\{v_{1}, v_{2}\right\}, & \mathbb{A}_{3}=\{w\}, & \mathbb{A}_{4}=\left\{s_{1}, s_{2}\right\}, \\
\mathbb{B}_{1}=\left\{s_{1}, s_{2}\right\}, & \mathbb{B}_{2}=\{\}, & \mathbb{B}_{3}=\{x\}, & \mathbb{B}_{4}=\{\}, \\
\mathbb{C}_{1}=\{\}, & \mathbb{C}_{2}=\left\{s_{1}, s_{2}\right\}, & \mathbb{C}_{3}=\left\{s_{1}, s_{2}\right\}, & \mathbb{C}_{4}=\{\}, \\
n_{1}=2, & n_{2}=0, & n_{3}=-1, & n_{4}=1, \\
\mathbb{A}_{5}=\{\}, & \mathbb{A}_{6}=\{x\}, & & \\
\mathbb{B}_{5}=\{\}, & \mathbb{B}_{6}=\{\}, & & \\
\mathbb{C}_{5}=\{\}, & \mathbb{C}_{6}=\{\}, & & \\
n_{5}=0, & n_{6}=0 . &
\end{array}
$$

Now we show how formulas (5), (6) and (8) give us the sought quiver polynomial. 
- Formula (5) claims that

$$
\begin{aligned}
{\left[\bar{\Omega}_{m}\right]=} & C_{\mathbb{A}_{1}, \mathbb{A}_{2}, \mathbb{A}_{3}, \mathbb{A}_{4}, \mathbb{A}_{5}, \mathbb{A}_{6}}^{\mathbb{K}_{2}, \mathbb{K}_{1}, \mathbb{K}_{3}, \mathbb{K}_{2}, \mathbb{K}_{1}, \mathbb{K}_{3}} \\
& \left(\frac{u^{2} s_{1} s_{2}\left(1-\frac{v_{1}}{v_{2}}\right)\left(1-\frac{s_{1}}{s_{2}}\right)\left(1-\frac{u}{s_{1}}\right)\left(1-\frac{u}{s_{2}}\right)\left(1-\frac{w}{x}\right)}{w\left(1-\frac{v_{1}}{s_{1}}\right)\left(1-\frac{v_{2}}{s_{1}}\right)\left(1-\frac{v_{1}}{s_{2}}\right)\left(1-\frac{v_{2}}{s_{2}}\right)\left(1-\frac{w}{s_{1}}\right)\left(1-\frac{w}{s_{2}}\right)}\right) .
\end{aligned}
$$

Computer calculation shows that the rational function in (9) is equal to $-u^{3}+$ $u^{2} s_{2}+u^{2} v_{2}+$ fractions. Hence

$$
\left[\bar{\Omega}_{m}\right]=-c_{3}^{(2)}+c_{2}^{(2)} c_{1}^{(2)}+c_{2}^{(2)} c_{1}^{(1)}
$$

where the upper index ${ }^{(i)}$ refers to the bundle $\mathcal{M}_{i}^{*}-\mathcal{E}_{i}^{*}$.

- Formula (6) claims that

$$
\begin{aligned}
{\left[\bar{\Omega}_{m}\right]=} & \boldsymbol{\Delta}_{\mathbb{A}_{1}, \mathbb{A}_{2}, \mathbb{A}_{3}, \mathbb{A}_{4}, \mathbb{A}_{5}, \mathbb{A}_{6}}^{\mathbb{K}_{2}, \mathbb{K}_{1}, \mathbb{K}_{3}, \mathbb{K}_{2}, \mathbb{K}_{1}, \mathbb{K}_{3}} \\
& \left(\frac{u^{2} s_{1} s_{2}\left(1-\frac{u}{s_{1}}\right)\left(1-\frac{u}{s_{2}}\right)\left(1-\frac{w}{x}\right)}{w\left(1-\frac{v_{1}}{s_{1}}\right)\left(1-\frac{v_{2}}{s_{1}}\right)\left(1-\frac{v_{1}}{s_{2}}\right)\left(1-\frac{v_{2}}{s_{2}}\right)\left(1-\frac{w}{s_{1}}\right)\left(1-\frac{w}{s_{2}}\right)}\right),
\end{aligned}
$$

cf. also Sect. 5.3. This is further equal to

$\boldsymbol{\Delta}_{\mathbb{A}_{1}, \mathbb{A}_{2}, \mathbb{A}_{3}, \mathbb{A}_{4}, \mathbb{A}_{5}, \mathbb{A}_{6}}^{\mathbb{K}_{2}, \mathbb{K}_{1}, \mathbb{K}_{3}, \mathbb{K}_{2}, \mathbb{K}_{1}, \mathbb{K}_{3}}\left(w u^{2}-2 u^{3}+2 u^{2} v_{1}+u^{2} s_{1}+\frac{s_{2}}{s_{1}} w u^{2}-\frac{s_{2}}{s_{1}} u^{3}+\frac{s_{2}}{s_{1}} u^{2} v_{1}+\cdots\right)$,

where the sign "..." means terms whose $\boldsymbol{\Delta}$-image is 0 . Hence we have

$$
\begin{aligned}
{\left[\bar{\Omega}_{m}\right]=} & \Delta_{1}^{(3)} \Delta_{2}^{(2)}-2 \Delta_{3}^{(2)}+2 \Delta_{2}^{(2)} \Delta_{1}^{(1)}+\Delta_{-1,1}^{(2)} \Delta_{1}^{(3)} \Delta_{2}^{(2)}+\Delta_{2}^{(2)} \Delta_{1}^{(2)} \\
& -\Delta_{-1,1}^{(2)} \Delta_{3}^{(2)}+\Delta_{-1,1}^{(2)} \Delta_{2}^{(2)} \Delta_{1}^{(1)} \\
= & \Delta_{1}^{(1)} \Delta_{2}^{(2)}-\Delta_{3}^{(2)}+\Delta_{2}^{(2)} \Delta_{1}^{(2)}
\end{aligned}
$$

which is equal to (10).

- Formula (8) claims

$$
\begin{aligned}
{\left[\bar{\Omega}_{m}\right]=} & \boldsymbol{\Delta}_{\left\{u, s_{1}, s_{2}\right\},\left\{v_{1}, v_{2}\right\},\{w, x\}}^{\mathbb{K}_{2}, \quad \mathbb{K}_{3},} \mathbb{K}_{3} \\
& \left(\frac{u^{2} s_{1} s_{2}}{w\left(1-\frac{v_{1}}{s_{1}}\right)\left(1-\frac{v_{2}}{s_{1}}\right)\left(1-\frac{v_{1}}{s_{2}}\right)\left(1-\frac{v_{2}}{s_{2}}\right)\left(1-\frac{w}{s_{1}}\right)\left(1-\frac{w}{s_{2}}\right)}\right),
\end{aligned}
$$

cf. also Sect. 5.3. This is further equal to

$$
\boldsymbol{\Delta}_{\left\{u, s_{1}, s_{2}\right\},\left\{v_{1}, v_{2}\right\},\{w, x\}}^{\mathbb{K}_{2},} \underset{\mathbb{K}_{3}}{\mathbb{K}_{1}}\left(w u^{2}+2 u^{2} v_{1}+\frac{s_{2}}{s_{1}} u^{2} v_{1}+\frac{s_{2}}{s_{1}} w u^{2}+u^{2} s_{1}+\cdots\right),
$$

where the sign "..." means terms whose $\boldsymbol{\Delta}$-image is 0 . Hence we have

$$
\left[\bar{\Omega}_{m}\right]=\Delta_{1}^{(3)} \Delta_{2}^{(2)}+2 \Delta_{2}^{(2)} \Delta_{1}^{(1)}+\Delta_{2,-1,1}^{(2)} \Delta_{1}^{(1)}+\Delta_{2,-1,1}^{(2)} \Delta_{1}^{(3)}+\Delta_{2,1}^{(2)}
$$




$$
=\Delta_{2}^{(2)} \Delta_{1}^{(1)}+\Delta_{2,1}^{(2)},
$$

which is equal to (10) - to see this, observe that $\Delta_{2,1}=\Delta_{2} \Delta_{1}-\Delta_{3}$.

Remark 6.1 Consider the rational function in the argument of (9). Observe that the $x$ variable only appears in the factor

$$
\left(1-\frac{w}{x}\right)=1+\frac{1}{x}(\cdots) .
$$

This means that the integer part of this rational function is the same as the integer part of it without this factor. Hence this factor can be dropped without changing the value of (9). Similar simplifications play an essential role in [20].

\section{Proof of Theorem 5.1}

Notation Let $\mathcal{E}^{e} \rightarrow X$ be a vector bundle over $X$ (the upper index is optional, it shows the rank of the bundle). The pull-back of this bundle along a map $\rho: Y \rightarrow X$ is denoted by $\rho^{*} \mathcal{E} \rightarrow Y$, or simply by $\mathcal{E} \rightarrow Y$. The class $c_{n}(\mathcal{E})$ may mean the $n$th Chern class of $\mathcal{E} \rightarrow X$ or the $n$th Chern class of $\mathcal{E} \rightarrow Y$, depending on context.

\subsection{Gysin map in iterated residue form}

The usual description of Gysin maps of Grassmannian (or flag) bundles is either via equivariant localization, or divided difference operations, or the combinatorics of partitions. The idea of studying these Gysin maps in iterated residue form is due to Bérczi and Szenes [2, Prop. 6.4]. The formulas in their natural generality are developed by Kazarian [22]. The following lemma is a special case of [22]. However, this particular statement is easily seen to be equivalent to the usual combinatorial description of the Gysin map $\pi_{*}$, see e.g. [19, Prop. in Sect. 4.1].

Lemma 7.1 Let $\mathcal{E}_{1}^{e_{1}}$ and $\mathcal{E}_{2}^{e_{2}}$ be vector bundles over $X$, and consider the Grassmann bundle $\pi: \mathrm{Gr}_{e_{2}-q} \mathcal{E}_{2} \rightarrow X$ of the bundle $\mathcal{E}_{2}$. The fiber of $\pi$ is $\mathrm{Gr}_{e_{2}-q} \mathbb{C}^{e_{2}}$, the Grassmannian of $e_{2}-q$-dimensional linear subspaces of $\mathbb{C}^{e_{2}}$. Let $S^{e_{2}-q} \rightarrow \mathcal{E}_{2} \rightarrow Q^{q}$ be the tautological exact sequence of bundles over $\mathrm{Gr}_{e_{2}-q} \mathcal{E}_{2}$. Let $\omega_{1}, \ldots, \omega_{q}$ be the Chern roots of $Q$, and let $\epsilon_{1}, \ldots, \epsilon_{e_{1}}$ be the Chern roots of $\mathcal{E}_{1}$. Let $f$ be a symmetric polynomial in $q$ variables. Then

$$
\pi_{*}\left(f\left(\omega_{1}, \ldots, \omega_{q}\right) \prod_{i=1}^{q} \prod_{j=1}^{e_{1}}\left(\omega_{i}-\epsilon_{j}\right)\right)=\boldsymbol{\Delta}_{\left\{v_{1}, \ldots, v_{q}\right\}}^{c_{n}\left(\mathcal{E}_{1}^{*}-\mathcal{E}_{2}^{*}\right)}\left(f\left(v_{1}, \ldots, v_{q}\right) \prod_{j=1}^{q} v_{j}^{e_{1}-e_{2}+q}\right) .
$$

\subsection{One step resolution in iterated residue form}

Consider a $\mathcal{Q}$-bundle over the space $X$, by which we now mean not only a collection of vector bundles $\mathcal{E}_{j}^{e_{j}}$ for $j \in \mathcal{Q}_{0}$, but also a vector bundle map $\phi_{a}: \mathcal{E}_{t(a)} \rightarrow \mathcal{E}_{h(a)}$ for every $a \in \mathcal{Q}_{1}$. Denote this $\mathcal{Q}$-bundle by $\left(\mathcal{E}_{j}, \phi_{a}\right) \rightarrow X$. 
Let us choose $i \in \mathcal{Q}_{0}$ and $q \in\left\{0,1, \ldots, e_{i}\right\}$. The numbers $i$ and $q$ will be fixed for the rest of the section. Following Reineke [29] (see also [5, 28]) we will describe a construction

$$
\left(\mathcal{E}_{j}, \phi_{a}\right) \rightarrow X \stackrel{R_{i}^{q}}{\longrightarrow}\left(\tilde{\mathcal{E}}_{j}, \tilde{\phi}_{a}\right) \rightarrow \tilde{X},
$$

and a map $\rho: \tilde{X} \rightarrow X$.

Let $\pi: \mathrm{Gr}_{e_{i}-q} \mathcal{E}_{i} \rightarrow X$ be the Grassmann bundle of $\mathcal{E}_{i}$. Over this space there is the tautological exact sequence $0 \rightarrow S \rightarrow \mathcal{E}_{i} \rightarrow Q \rightarrow 0$. Recall that $\mathcal{M}_{j}=\bigoplus_{k \in T(j)} \mathcal{E}_{k}$. Consider the zero scheme $\tilde{X}$ of the composition map $\mathcal{M}_{i} \rightarrow \mathcal{E}_{i} \rightarrow Q$, and its embed$\operatorname{ding} \iota: \tilde{X} \rightarrow \operatorname{Gr}_{e_{i}-q} \mathcal{E}_{i}$. The $\mathcal{Q}$-bundle $\left(\mathcal{E}_{j}, \phi_{a}\right)$ over $X$ induces a $\mathcal{Q}$-bundle $\left(\tilde{\mathcal{E}}_{j}, \tilde{\phi}_{a}\right)$ over $\tilde{X}$ as follows:

- $\tilde{\mathcal{E}}_{j}=\mathcal{E}_{j}$ if $j \neq i$, and $\tilde{\mathcal{E}}_{i}=S$;

- $\tilde{\phi}_{a}=\phi_{a}$ if $h(a) \neq i, t(a) \neq i$;

- $\tilde{\phi}_{a}$ is the composition $S \rightarrow \mathcal{E}_{i} \stackrel{\phi_{a}}{\longrightarrow} \mathcal{E}_{h(a)}$ if $t(a)=i$;

- $\tilde{\phi}_{a}: \mathcal{E}_{j} \rightarrow S$ is the unique lifting of $\phi_{a}: \mathcal{E}_{j} \rightarrow \mathcal{E}_{i}$ over $\tilde{X}$, if $h(a)=i$.

Let $\rho=\pi \circ \iota: \tilde{X} \rightarrow X$.

Our goal now is a formula for the Gysin map $\rho_{*}$. Consider $p$ sets of variables

$$
\mathbb{A}_{1}=\left\{u_{11}, \ldots, u_{1 r_{1}}\right\}, \quad \mathbb{A}_{2}=\left\{u_{21}, \ldots, u_{2 r_{2}}\right\}, \quad \ldots, \quad \mathbb{A}_{p}=\left\{u_{p 1}, \ldots, u_{p r_{p}}\right\},
$$

and the numbers $i_{1}, i_{2}, \ldots, i_{p} \in \mathcal{Q}_{0}$. Set $\tilde{\mathcal{M}}_{j}=\bigoplus_{k \in T(j)} \tilde{\mathcal{E}}_{k}$. For $j \in \mathcal{Q}_{0}$ let

$$
\tilde{\mathbb{K}}_{j}=\left(c_{n}\left(\tilde{\mathcal{M}}_{j}^{*}-\tilde{\mathcal{E}}_{j}^{*}\right)\right), \quad \mathbb{K}_{j}=\left(c_{n}\left(\mathcal{M}_{j}^{*}-\mathcal{E}_{j}^{*}\right)\right) .
$$

In particular, if $j \neq i, j \notin H(i)$ then $\tilde{\mathbb{K}}_{j}=\mathbb{K}_{j}$. Let $\mathbb{A}_{0}=\left\{v_{1}, \ldots, v_{q}\right\}$ and $i_{0}=i$.

Proposition 7.2 For the Gysin map $\rho_{*}: H^{*}(\tilde{X}) \rightarrow H^{*}(X)$ we have

$$
\begin{aligned}
\rho_{*} & \left(\boldsymbol{\Delta}_{\mathbb{A}_{1}, \ldots, \mathbb{A}_{p}}^{\tilde{\mathbb{K}}_{i_{1}}, \ldots, \tilde{\mathbb{K}}_{i_{p}}}\left(f\left(u_{k s}\right)\right)\right) \\
& =\boldsymbol{\Delta}_{\mathbb{A}_{0}, \mathbb{A}_{1}, \ldots, \mathbb{A}_{p}}^{\mathbb{K}_{i_{0}}, \mathbb{K}_{i_{1}}, \ldots, \mathbb{K}_{i p}}\left(f\left(u_{k s}\right) \prod_{v \in \mathbb{A}_{0}} v^{\mathrm{rk} \mathcal{M}_{i}-\mathrm{rk} S} \cdot \prod_{v \in \mathbb{A}_{0}} \frac{\prod_{k \geq 1, i_{k}=i} \prod_{k \in \mathbb{A}_{k}}\left(1-\frac{v}{x}\right)}{\prod_{k \geq 1, i_{k} \in H(i)} \prod_{x \in \mathbb{A}_{k}}\left(1-\frac{v}{x}\right)}\right) .
\end{aligned}
$$

Proof During the proof we will use the following notation:

$$
\begin{aligned}
& P_{j}=\prod_{k \geq 1, i_{k}=j} \prod_{j \in \mathbb{A}_{k}} \sum_{n=0}^{\infty} \frac{c_{n}\left(\mathcal{M}_{j}^{*}-\mathcal{E}_{j}^{*}\right)}{x^{n}}, \\
& \tilde{P}_{j}=\prod_{k \geq 1, i_{k}=j} \prod_{j \in \mathbb{A}_{k}} \sum_{n=0}^{\infty} \frac{c_{n}\left(\tilde{\mathcal{M}}_{j}^{*}-\tilde{\mathcal{E}}_{j}^{*}\right)}{x^{n}}, \\
& P_{j}^{\prime}=\prod_{k \geq 0, i_{k}=j} \prod_{x \in \mathbb{A}_{k}} \sum_{n=0}^{\infty} \frac{c_{n}\left(\mathcal{M}_{j}^{*}-\mathcal{E}_{j}^{*}\right)}{x^{n}} .
\end{aligned}
$$


We will study the relations among $P_{j}, \tilde{P}_{j}$, and $P_{j}^{\prime}$ using the formal identity

$$
\sum_{n=0}^{\infty} \frac{c_{n}\left(\mathcal{E}^{*}-\mathcal{F}^{*}\right)}{x^{n}}=\frac{\prod_{l}\left(1-\frac{\epsilon_{l}}{x}\right)}{\prod_{l}\left(1-\frac{\phi_{l}}{x}\right)}
$$

for the Chern roots $\epsilon_{l}$ (resp. $\phi_{l}$ ) of the bundle $\mathcal{E}$ (resp. $\mathcal{F}$ ). Namely, we have

- $\tilde{P}_{j}=P_{j}$ unless $j=i$ or $j=\in H(i)$.

- Let the Chern roots of $\tilde{\mathcal{M}}_{i}=\mathcal{M}_{i}$ be $\mu_{l}$, let the Chern roots of $\tilde{\mathcal{E}}_{i}=S$ be $\sigma_{l}$, let the Chern roots of $\mathcal{E}_{i}$ be $\epsilon_{l}$, and let the Chern roots of $Q$ be $\omega_{l}$. Then

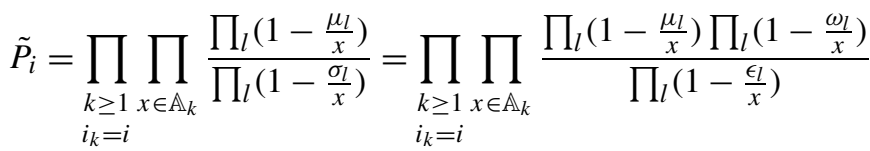

$$
\begin{aligned}
& =P_{i} \prod_{\substack{k \geq 1 \\
i_{k}=i}} \prod_{\substack{x \in \mathbb{A}_{k} \\
l}}\left(1-\frac{\omega_{l}}{x}\right) .
\end{aligned}
$$

- Let $j \in H(i)$. Let the Chern roots of $\tilde{M}_{j}$ be $\nu_{l}$, the Chern roots of $\mathcal{E}_{j}$ be $\kappa_{l}$, and the Chern roots of $\mathcal{M}_{j}$ be $\gamma_{l}$. We have

$$
\begin{aligned}
& \tilde{P}_{j}=\prod_{\substack{k \geq 1 \\
i_{k}=j}} \prod_{\substack{x \in \mathbb{A}_{k} \\
\prod_{l}\left(1-\frac{\kappa_{l}}{x}\right)}}^{\prod_{l}\left(1-\frac{\nu_{l}}{x}\right)} \prod_{\substack{k \geq 1 \\
i_{k}=j}} \prod_{\substack{x \in \mathbb{A}_{k} \\
\prod_{l}\left(1-\frac{\kappa_{l}}{x}\right) \prod_{l}\left(1-\frac{\omega_{l}}{x}\right)}} \\
& =P_{j} \prod_{\substack{k \geq 1 \\
i_{k}=j}} \prod_{\substack{x \in \mathbb{A}_{k} \\
l}} \frac{1}{\left(1-\frac{\omega_{l}}{x}\right)} .
\end{aligned}
$$

- $P_{j}^{\prime}=P_{j}$ unless $j=i$.

- $P_{i}^{\prime}=P_{i} \prod_{v \in \mathbb{A}_{0}} \sum_{n=0}^{\infty} \frac{c_{n}\left(\mathcal{M}_{i}^{*}-\mathcal{E}_{i}^{*}\right)}{v^{n}}$.

Now we may start the proof. Setting

$$
g\left(u_{k s}\right)=f\left(u_{k s}\right) \prod_{k=1}^{p} \prod_{1 \leq l<j \leq r_{k}}\left(1-\frac{u_{k l}}{u_{k j}}\right)
$$

we may use Lemmas 2.5 and 2.6 to write

$$
z=\boldsymbol{\Delta}_{\mathbb{A}_{1}, \ldots, \mathbb{A}_{p}}^{\tilde{\mathbb{K}}_{i_{1}}, \ldots, \tilde{\mathbb{K}}_{i_{p}}}\left(f\left(u_{k s}\right)\right)=\left\{g\left(u_{k s}\right) \cdot \prod_{j=1}^{N} \tilde{P}_{j}\right\}_{u_{k s}},
$$


where $\{\cdots\}_{u_{k s}}$ means the constant term in the $u_{k s}$ variables. Using the relations between $P_{j}$ and $\tilde{P}_{j}$, this is further equal to

$$
\left\{g\left(u_{k s}\right) \frac{\prod_{k \geq 1, i_{k}=i} \prod_{x \in \mathbb{A}_{k}} \prod_{l}\left(1-\frac{\omega_{l}}{x}\right)}{\prod_{k \geq 1, i_{k} \in H(i)} \prod_{x \in \mathbb{A}_{k}} \prod_{l}\left(1-\frac{\omega_{l}}{x}\right)} \cdot \prod_{j=1}^{N} P_{j}\right\}_{u_{k s}} .
$$

This class is an expression in Chern roots of bundles, all of which pull back via $\iota$. Hence, the adjunction formula $\iota_{*}\left(\iota^{*}(h)\right)=h \cdot \iota_{*}(1)$ of the Gysin map implies that $\iota_{*}(z)$ is the same expression as $(14)$, times

$$
\iota_{*}(1)=e\left(\operatorname{Hom}\left(\mathcal{M}_{i}, Q\right)\right)=\prod_{l=1}^{q} \prod_{j=1}^{\mathrm{rk} \mathcal{M}_{i}}\left(\omega_{l}-\mu_{j}\right) .
$$

We can apply Lemma 7.1 to obtain

$$
\begin{aligned}
\rho_{*}(z)= & \pi_{*} \iota_{*}(z) \\
= & \boldsymbol{\Delta}_{\mathbb{A}_{0}}^{\mathbb{K}_{i_{0}}}\left(\prod_{v \in \mathbb{A}_{0}} v^{\mathrm{rk} \mathcal{M}_{i}-\mathrm{rk} S}\right. \\
& \left.\times\{\underbrace{g\left(u_{k s}\right) \frac{\prod_{k \geq 1, i_{k}=i} \prod_{x \in \mathbb{A}_{k}} \prod_{v \in \mathbb{A}_{0}}\left(1-\frac{v}{x}\right)}{\prod_{k \geq 1, i_{k} \in H(i)} \prod_{x \in \mathbb{A}_{k}} \prod_{v \in \mathbb{A}_{0}}\left(1-\frac{v}{x}\right)} \prod_{j=1}^{N} P_{j}}_{\boldsymbol{\alpha}}\}_{u_{k s}}\right) .
\end{aligned}
$$

Using Lemmas 2.5 and 2.6 and the comparison of $P_{i}$ with $P_{i}^{\prime}$, this is further equal to

$$
\begin{aligned}
& \left\{\prod_{v \in \mathbb{A}_{0}} v^{\mathrm{rk} \mathcal{M}_{i}-\mathrm{rk} S} \prod_{l<l^{\prime}}\left(1-\frac{v_{l}}{v_{l^{\prime}}}\right)\{\boldsymbol{\alpha}\}_{u_{k s}} \prod_{v \in \mathbb{A}_{0}} \sum_{n=1}^{\infty} \frac{c_{n}\left(\mathcal{M}_{i}^{*}-\mathcal{E}_{i}^{*}\right)}{v^{n}}\right\}_{v_{l}} \\
& =\left\{\prod_{v \in \mathbb{A}_{0}} v^{\mathrm{rk} \mathcal{M}_{i}-\mathrm{rk} S} \prod_{l<l^{\prime}}\left(1-\frac{v_{l}}{v_{l^{\prime}}}\right) \boldsymbol{\otimes} \prod_{v \in \mathbb{A}_{0}} \sum_{n=0}^{\infty} \frac{c_{n}\left(\mathcal{M}_{i}^{*}-\mathcal{E}_{i}^{*}\right)}{v^{n}}\right\}_{u_{k s}, v_{l}} \\
& =\left\{\prod_{v \in \mathbb{A}_{0}} v^{\mathrm{rk} \mathcal{M}_{i}-\mathrm{rk} S} \prod_{l<l^{\prime}}\left(1-\frac{v_{l}}{v_{l^{\prime}}}\right) g\left(u_{k s}\right)\right. \\
& \left.\quad \times \frac{\prod_{k \geq 1, i_{k}=i} \prod_{x \in \mathbb{A}_{k}} \prod_{v \in \mathbb{A}_{0}}\left(1-\frac{v}{x}\right)}{\prod_{k \geq 1, i_{k} \in H(i)} \prod_{x \in \mathbb{A}_{k}} \prod_{v \in \mathbb{A}_{0}}\left(1-\frac{v}{x}\right)} \cdot \prod_{j=1}^{N} P_{j}^{\prime}\right\}_{u_{k s}, v_{l}} .
\end{aligned}
$$

Now, using Lemmas 2.5 and 2.6 again, backwards, we obtain

$\rho_{*}(z)$

$$
=\boldsymbol{\Delta}_{\mathbb{A}_{0}, \mathbb{A}_{1}, \ldots, \mathbb{A}_{p}}^{\mathbb{K}_{i}, \mathbb{K}_{i_{1}}, \ldots, \mathbb{K}_{i p}}\left(\prod_{v \in \mathbb{A}_{0}} v^{\mathrm{rk} \mathcal{M}_{i}-\mathrm{rk} S} f\left(u_{k s}\right) \frac{\prod_{k \geq 1, i_{k}=i} \prod_{x \in \mathbb{A}_{k}} \prod_{v \in \mathbb{A}_{0}}\left(1-\frac{v}{x}\right)}{\prod_{k \geq 1, i_{k} \in H(i)} \prod_{x \in \mathbb{A}_{k}} \prod_{v \in \mathbb{A}_{0}}\left(1-\frac{v}{x}\right)}\right),
$$

what we wanted to prove. 


\subsection{Iterated application of Proposition 7.2}

We are ready to prove Theorem 5.1. Let $\mathcal{Q}$ be a quiver of Dynkin type, and let $\Omega_{m}$ be an orbit of a quiver representation. Let $\boldsymbol{i}, \boldsymbol{r}$ be a resolution pair for $\Omega_{m}$.

Let $\left(\mathcal{E}_{j}, \phi_{a}\right) \rightarrow X$ be a $\mathcal{Q}$-bundle over the space $X$. Define the degeneracy locus

$$
\Omega\left(\mathcal{E}_{j}, \phi_{a}\right)=\left\{x \in X:\left(E_{j}, \phi_{a}\right)_{x} \in \bar{\Omega}_{m}\right\} .
$$

Theorem 7.3 (Reineke [29, Theorem 2.2]) Let $\tilde{X}$ be the base space of the bundle obtained by applying $R_{i_{1}}^{r_{1}}$, then $R_{i_{1}}^{r_{1}}$, etc., up to $R_{i_{p}}^{r_{p}}$ to the $\mathcal{Q}$-bundle $\left(\mathcal{E}_{j}, \phi_{a}\right) \rightarrow X$. The map

$$
\rho_{i_{1}}^{r_{1}} \circ \rho_{i_{2}}^{r_{2}} \circ \cdots \circ \rho_{i_{p}}^{r_{p}}: \tilde{X} \rightarrow X
$$

is a resolution of $\bar{\Omega}\left(\mathcal{E}_{j}, \phi_{a}\right)$.

We can apply Theorem 7.3 to maps between the universal vector bundles described in Sect. 4.2, and we get

$$
\left[\bar{\Omega}_{m}\right]=\left(\rho_{i_{1}}^{r_{1}} \circ \rho_{i_{2}}^{r_{2}} \circ \cdots \circ \rho_{i_{p}}^{r_{p}}\right)_{*}(1)=\rho_{i_{1 *}}^{r_{1}}\left(\rho_{i_{2} *}^{r_{2}}\left(\cdots\left(\rho_{i_{p} *}^{r_{p}}\left(\boldsymbol{\Delta}_{\emptyset}^{\emptyset}(1)\right)\right) \cdots\right)\right) .
$$

If we apply the statement of Theorem 7.2 at each application of $\rho_{*}$, we get the statement of Theorem 5.1 .

\section{On Buch's conjecture}

For an alphabet $\mathbb{X}=\left(x_{1}, \ldots, x_{r}\right)$ and a sequence of integers $\lambda \in \mathbb{Z}^{r}$ let $\mathbb{X}^{\lambda}$ be $\prod_{i=1}^{r} x_{i}^{\lambda(i)}$. We can reformulate Buch's conjecture on the positivity of cohomological quiver coefficients, in the terminology of the present paper.

Conjecture 8.1 (Buch [5]) Let $\mathcal{Q}$ be a Dynkin quiver, $\left(e_{1}, \ldots, e_{N}\right)$ a dimension vector, and $\Omega_{m}$ an orbit of the corresponding representation. Let $\mathbb{X}_{i}$ be alphabets of cardinality $e_{i}, i=1, \ldots, N$. There exists a function

$$
f=\sum c_{\left(\lambda_{1}, \ldots, \lambda_{N}\right)} \mathbb{X}_{1}^{\lambda_{1}} \cdots \mathbb{X}_{N}^{\lambda_{N}}
$$

where the summation runs for $N$-tuples of partitions, and $c_{\left(\lambda_{1}, \ldots, \lambda_{N}\right)} \geq 0$, such that

$$
\left[\bar{\Omega}_{m}\right]=\boldsymbol{\Delta}_{\mathbb{X}_{1}, \ldots, \mathbb{X}_{N}}^{\mathbb{K}_{1}, \ldots \mathbb{K}_{N}}\left(f\left(\mathbb{X}_{1}, \ldots, \mathbb{X}_{N}\right)\right)
$$

In (8) we gave an $f$ satisfying (15). However, that particular $f$ uses non-partition exponents as well, and may not satisfy the positivity condition. The challenge in proving Buch's conjecture is to find an $f$ " $\boldsymbol{\Delta}$-equivalent" to the formula in (8) but satisfying the partition and positivity conditions. Initial results in this direction are in [20].

Acknowledgements The author thanks J. Allman, A.S. Buch, L. Fehér, R. Kaliszewski, M. Kazarian, and A. Szenes for valuable discussions on the topic, as well as the hospitality of MPIM Bonn and the University of Geneva. The author was partially supported by NSF grant DMS-1200685. 


\section{References}

1. Abeasis, S., Del Fra, A.: Degenerations for the representations of an equioriented quiver of type $A_{m}$. J. Algebra 93, 376-412 (1985)

2. Bérczi, G., Szenes, A.: Thom polynomials of Morin singularities. Ann. Math. (2) 175(2), 567-629 (2012)

3. Bertein, I.N., Gel'fand, I.M., Ponomarev, V.A.: Coxeter functors and Gabriel's theorem. Usp. Mat. Nauk 28, 19-33 (1973)

4. Buch, A.S.: Grothendieck classes of quiver varieties. Duke Math. J. 115(1), 75-103 (2002)

5. Buch, A.S.: Quiver coefficients of Dynkin type. Mich. Math. J. 57, 93-120 (2008)

6. Buch, A.S., Fulton, W.: Chern class formulas for quiver varieties. Invent. Math. 135, 665-687 (1999)

7. Buch, A.S., Rimányi, R.: A formula for non-equioriented quiver orbits of type A. J. Algebr. Geom. 16, 531-546 (2007)

8. Buch, A.S., Kresch, A., Tamvakis, H., Yong, A.: Schubert polynomials and quiver formulas. Duke Math. J. 122, 125-143 (2004)

9. Buch, A.S., Fehér, L., Rimányi, R.: Positivity of quiver coefficients through Thom polynomials. Adv. Math. 197, 306-320 (2005)

10. Buch, A.S., Sottile, F., Yong, A.: Quiver coefficients are Schubert structure constants. Math. Res. Lett. 12, 567-574 (2005)

11. Edidin, D., Graham, W.: Characteristic classes in the Chow ring. J. Algebr. Geom. 6(3), 431-443 (1997)

12. Fehér, L., Rimányi, R.: Classes of degeneracy loci for quivers: the Thom polynomial point of view. Duke Math. J. 114(2), 193-213 (2002)

13. Fehér, L.M., Rimányi, R.: Calculation of Thom polynomials and other cohomological obstructions for group actions. In: Gaffney, T., Ruas, M. (eds.) Real and Complex Singularities (Sao Carlos, 2002). Contemp. Math., vol. 354, pp. 69-93. AMS, Providence (2004)

14. Fehér, L., Rimányi, R.: On the structure of Thom polynomials of singularities. Bull. Lond. Math. Soc. 39, 541-549 (2007)

15. Fehér, L., Rimányi, R.: Thom series of contact singularities. Ann. Math. 176(3), 1381-1426 (2012)

16. Fomin, S.V., Gelfand, S., Postnikov, A.: Quantum Schubert polynomials. J. Am. Math. Soc. 10, 565596 (1997)

17. Fulton, W.: Universal Schubert polynomials. Duke Math. J. 96, 575-594 (1999)

18. Fulton, W.: Equivariant cohomology in algebraic geometry. Eilenberg lectures, Columbia University (2007). http://www.math.washington.edu/ dandersn/eilenberg/

19. Fulton, W., Pragacz, P.: Schubert Varieties and Degeneracy Loci. Lect. Notes Math., vol. 1689. Springer, Berlin (1998)

20. Kaliszewski, R.: Structure of quiver polynomials and Schur positivity. Ph.D. thesis, UNC (2013)

21. Kazarian, M.É.: Characteristic classes of singularity theory. In: The Arnold-Gelfand Mathematical Seminars, pp. 325-340. Birkhäuser Boston, Cambridge (1997)

22. Kazarian, M.: Gysin homomorphism and degeneracies (2010, preprint)

23. Kazarian, M.: Non-associative Hilbert scheme and Thom polynomials (2010, preprint)

24. Knutson, A., Shimozono, M.: Kempf collapsing and quiver loci (2006, preprint)

25. Knutson, A., Miller, E., Shimozono, M.: Four positive formulae for type A quiver polynomials. Invent. Math. 166(2), 229-325 (2006)

26. Kontsevich, M., Soibelman, Y.: Cohomological Hall algebra, exponential Hodge structures and motivic Donaldson-Thomas invariants (2010). arXiv:1006.2706

27. Miller, E., Strumfels, B.: Combinatorial Commutative Algebra. Graduate Texts in Mathematics, vol. 227. Springer, New York (2005)

28. Porteous, I.R.: Simple singularities of maps. In: Proceedings of Liverpool SingularitiesSymposium I (1969/1970). Lect. Notes Math., vol. 192, pp. 286-307. Springer, Berlin (1971)

29. Reineke, M.: Quivers, desingularizations and canonical bases. In: Studies in Memory of Issai Schur (Chevaleret/Rehovot, 2000). Prog. Math., vol. 210, pp. 325-344. Birkhäuser, Basel (2003)

30. Rimányi, R.: Equivariant fundamental classes and the structure of COHA for Dynkin quivers (2013, preprint) 